Classification

Physics Abstracts

$79.20 \mathrm{~K}-82.80-91.65$

\title{
A study of silicon ELNES in nesosilicates
}

\author{
David W. McComb $\left({ }^{1}\right)$, Poul L. Hansen $\left({ }^{2}\right)$ and Rik Brydson $\left({ }^{3, *}\right)$
}

( $\left.{ }^{1}\right)$ Microstructural Physics Group, Cavendish Laboratory, Madingley Rd., Cambridge CB3 OHE, G.B.

$\left({ }^{2}\right)$ Laboratory of Applied Physics, Technical University of Denmark, 2800 Lyngby, Denmark

$\left({ }^{3}\right)$ The Blackett Laboratory, Imperial College, Prince Consort Rd., London SW7 2B2, G.B.

(Received November 04, 1991; accepted November 07, 1991)

\begin{abstract}
Si $\mathrm{L}_{2,3}$-absorption edges have been collected using EELS from four nesosilicates; Forsterite, Fayalite, Phenacite and Willemite. Nesosilicates, also known as orthosilicates or island silicates, are the simplest materials to be found in the silicate mineral class, consisting of isolated tetrahedral $\mathrm{SiO}_{4}$ groups. While many features in energy-loss spectra are common to all of the materials, remarkable extra intensity was observed for Forsterite. This appears to arise from distortion of the $\mathrm{SiO}_{4}$ tetrahedron; a hypothesis supported by XANES calculations. The concept of a simple fingerprint in near-edge structure analysis is questioned.
\end{abstract}

\section{Introduction.}

Electron energy-loss spectroscopy (EELS) carried out in the electron microscope is now a well established technique for the measurement of chemical composition with high spatial resolution [1]. The energy-loss near-edge structure (ELNES) of inner-shell ionisation edges can also be used to gain insight into the chemical and structural properties of the atom undergoing excitation.

At present, there are three different theoretical frameworks, recently reviewed by Brydson [2], within which ELNES can be interpreted. From a band structure point of view, ELNES is proportional to a symmetry projected local density of empty states (LDOES) [1]. The multiple scattering (MS) approach is a real space cluster method based on the interference between the outgoing electron wave of the ejected electron and the wave which is backscattered by the surrounding atoms [3]. Both methods incorporate an atomic transition matrix element between the initial and final states of the electron. The third approach to the interpretation of ELNES is to assign peaks to particular transitions between occupied and unoccupied states in the theoretical molecular orbital (MO) diagram of the species being investigated.

The band structure approach has been applied mainly to elemental or simple binary compounds, such as diamond [4] and silicon carbide [5], while the MS method has been used to interpret the ELNES of more complex structures, such as those found in minerals. Brydson et al. successfully modelled the ELNES in the minerals Rhodizite [6] and Chrysoberyl [7], and proposed

$\left(^{*}\right)$ Now at, University of Oxford, Dept. of Materials, Parks Rd., Oxford OX1 3PH, G.B. 
the existence of cation coordination fingerprints. MO calculations have been used to explain differences in X-ray absorption spectroscopy (XAS) and X-ray emission spectroscopy (XES) data from tetrahedrally coordinated ions in oxides [8], to explain the variation in ELNES from a series of compounds with silicon in an increasingly oxidised stare [9], and to interpret the ELNES of fullerite [10].

In this paper, the ELNES of the $\mathrm{Si}_{2,3}$ excitation from four nesosilicates are reported. In the materials studied - Forsterite, Fayalite, Willemite and Phenacite - the basic structural unit is an isolated $\mathrm{SiO}_{4}$ tetrahedron. $\mathrm{Si} \mathrm{L}, 3$ ELNES from the various allotropes of $\mathrm{SiO}_{2}$, which are all based on the same four-fold coordination as the nesosilicates, are almost identical, and it has been suggested that long- and medium-range order have little or no effect on the ELNES [11-13]. A systematic study of the $\mathrm{Si}_{2,3}$ ELNES in all of the major classes of silicates has been initiated and the present paper is the first of a series, although the results of a complimentary investigation of the silicon K-edge excitations have already been reported [14].

\section{Experimental details.}

The mineral samples were provided by the Mineralogical Museum in Copenhagen, Denmark, with the following reference numbers; Forsterite $-\mathrm{Mg}_{2} \mathrm{SiO}_{4}$ (1974.676), Fayalite $-\mathrm{Fe}_{2} \mathrm{SiO}_{4}$ (1907.133), Phenacite $-\mathrm{Be}_{2} \mathrm{SiO}_{4}$ (1974.791) and Willemite $-\mathrm{Zn}_{2} \mathrm{SiO}_{4}$ (1978.406). It should be noted that, the different cations aside, Forsterite and Fayalite are isostructural, as are Phenacite and Willemite. As discussed above, $\mathrm{SiO}_{4}$ tetrahedra are common to all four structures, but in Forsterite and Fayalite these are linked by metal cations octahedrally coordinated to oxygen, while in Phenacite and Willemite the cations are present in tetrahedral coordination.

The samples were crushed and ground with a mortar and pestle to a fine powder. The powder was suspended in ethanol and a drop of the suspension was placed on a holey carbon film covered copper grid and allowed to dry. Approximate chemical composition and crystal structure of the minerals were checked in the TEM using selected area diffraction and energy-dispersive X-ray (EDX) analysis. EELS was carried out in a VG HB501 dedicated STEM using a recently developed parallel recording system [15]. The spectra were acquired using an effective collection angle of $8.3 \mathrm{mrad}$ in image mode with an energy dispersion of about $0.2 \mathrm{eV}$ per channel, resulting in an energy resolution of approximately $0.5 \mathrm{eV}$. No attempt was made at this stage to investigate the effect of crystal orientation on the observed energy-loss spectrum.

\section{Results and discussion.}

The silicon $\mathrm{L}_{2,3}$-edges of Forsterite, (a), and Fayalite, (b), are shown in figure 1. A background of the form $A E^{-r}$ has been subtracted, but the spectra have not been deconvoluted with the valence loss spectra to remove contributions due to multiple energy losses. However, these do not influence the ELNES in the region of interest, which is within 10-15 eV of the ionisation threshold. The spectra were aligned by setting the position of the main peak, $\beta$, to $108.3 \mathrm{eV}$ as measured in $\mathrm{SiO}_{2}$ by Batson et al. [12]. The spectrum of Fayalite is almost identical to that of $\mathrm{SiO}_{2}$, with a $2 \mathrm{eV}$ separation between the pre-peak, $\alpha$, and the main peak, $\beta$. There is a distinct valley in the spectrum of Fayalite at $\beta^{\prime}$, but in that of Forsterite the valley has been completely filled.

Figure 2 shows the silicon $\mathrm{L}_{2,3}$-edges of Willemite, (a), and Phenacite, (b). The spectrum of Willemite is very similar to that of Fayalite, and this shape has been identified in a large range of silicates. In the spectrum of Phenacite the energy separation between the pre-peak and the main 


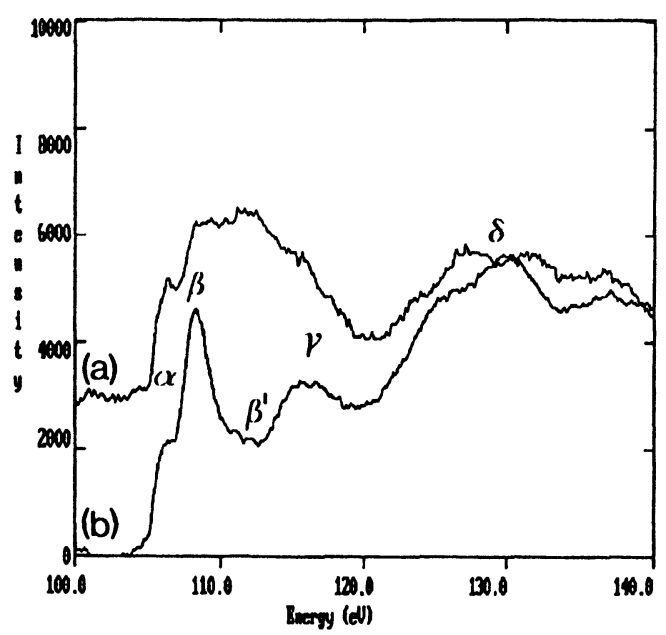

Fig. 1. - Silicon $\mathrm{L}_{2,3}$-edges from Forsterite $-\mathrm{Mg}_{2} \mathrm{SiO}_{4}$ (a), and Fayalite $-\mathrm{Fe}_{2} \mathrm{SiO}_{4}$ (b).

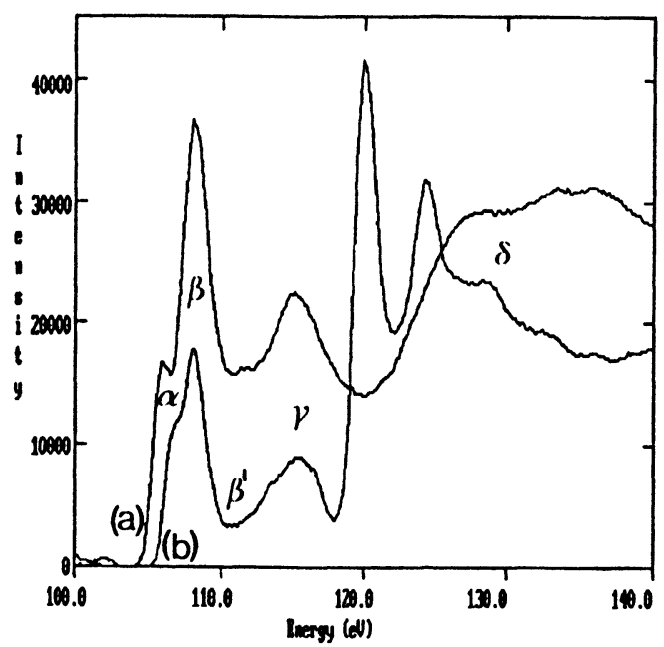

Fig. 2. - Silicon $\mathrm{L}_{2,3}$-edges from Willemite $-\mathrm{Zn}_{2} \mathrm{SiO}_{4}$ (a), and Phenacite $-\mathrm{Be}_{2} \mathrm{SiO}_{4}$ (b).

peak is only $1.0 \mathrm{eV}$, and the intensity ratio of the two peaks is $2: 3$, rather than $1: 2$ as observed in the other spectra. The Phenacite spectrum cannot be interpreted beyond $118 \mathrm{eV}$ due to the presence of strong features associated with the beryllium K-edge. In all of the spectra a large, broad peak, $\delta$, is observed approximately $22 \mathrm{eV}$ above the energy-loss of the main peak. This complex characteristic has contributions arising from multiple energy-losses, transitions to the continuum, and multiple scattering (shape) resonances, and will not be analysed in detail.

The dipole selection rule, $\Delta \ell= \pm 1$, applies approximately to the energy-loss spectrum in cases where, as in this study, a small collection aperture is used [1]. As a start towards understanding the $\mathrm{Si} \mathrm{L}_{2,3}$ ELNES, MO calculations for the $\mathrm{SiO}_{4}^{4-}$ cluster can be used to allocate the peaks in the spectra to transitions to specific molecular orbitals. On the basis of Tossel's calculations [8], 
peaks $\alpha$ and $\beta$ can be assigned to the molecular orbitals labelled $6 \mathrm{t}_{2}$ and $6 \mathrm{a}_{1}$, which exhibit $\mathrm{p}$ - and s-like symmetry, respectively. The next available empty states in the MO calculation are situated about $6.0 \mathrm{eV}$ above the lowest unoccupied $\mathrm{MO}$, and are designated $2 \mathrm{e}, 7 \mathrm{t}_{2}$ and $7 \mathrm{a}_{1}$ with $\mathrm{d}-\mathrm{d}$, $\mathrm{d}$ and s-like symmetry, respectively. This group of states corresponds approximately to the peak $\gamma$ in the energy-loss spectrum. At this point it is worth noting that a $\mathrm{p} \rightarrow \mathrm{p}$ transition is dipole-allowed in a tetrahedral molecule [21]. This arises as a result of the lack of a centre of inversion in the molecule, and means that the peaks observed in the $\mathrm{Si}_{2,3}$ ELNES may be associated with final states of s-, p- or d-like symmetry.

The MO calculations describe the empty levels and their ordering in the ground state, but changes in the energy level diagram in the excited state are not considered. Other workers have shown that the energy level diagram can be significantly altered by the presence of a core hole created by electron excitation. Therefore, it is advisable to compare the experimental data either with calculations in which the effect of the core hole has been accounted for, or with experimental data from other techniques. It is also well established that the energy separations of the MOs at higher energy are unreliable and it is misleading to place too much emphasis on the precise values.

The energy-loss spectra can be compared to the X-ray equivalent of ELNES i.e. X-ray absorption near-edge structure (XANES). Using XANES, Dehmer [16] has reported a silicon $\mathrm{L}_{2,3}$-edge in $\mathrm{SiO}_{2}$ which is similar to the ELNES of Fayalite and Willemite. By comparison with a detailed study of the XANES of $\mathrm{SF}_{6}$ and $\mathrm{Na}_{2} \mathrm{SiF}_{6}$, the peaks in the spectrum of $\mathrm{SiO}_{2}$ were given assignments by Dehmer [16] similar to those derived above from the Tossel MO calculations. However, the allocation of the first two peaks is reversed with respect to the MO calculation. Thus, the first peak in the spectrum is associated with an empty state of s-like symmetry, $a_{1}$, rather than p-like symmetry, $t_{2}$. In addition, Dehmer assigns the peak at $130 \mathrm{eV}$, corresponding to peak $\delta$ in the EELS data, to transitions to an unoccupied MO with d-like symmetry, and the XANES peak which aligns with the EELS peak $\gamma$ is associated with a d-like state, e.

It is difficult to decide which of these assignments is correct. In a description of the MO calculations, Tossel [8] indicates that the energy of the s-like $\left(6 \mathrm{a}_{1}\right)$ molecular orbital is sensitive to electronic effects, and it is perhaps not too surprising that its position may be altered by core hole effects. Thus, on the basis of the MO calculations by Tossel [8] and the X-ray absorption studies of Dehmer [16], the peaks in the EELS spectra of Fayalite and Willemite, and to a first approximation Forsterite and Phenacite, may be assigned to unoccupied MOs as follows: $\alpha-\mathrm{a}_{1}$ (s-like), $\beta-\mathrm{t}_{2}$ (p-like), $\gamma-$ primarily $\mathrm{e}\left(\mathrm{d}\right.$-like), and finally, $\delta-\mathrm{t}_{2}$ (d-like). This allocation is not as clear as might be desired, and does not simplify the interpretation of changes in the energy-loss spectra. For this reason, it was decided to model the EELS data using a XANES multiple scattering algorithm [3].

The structure in the silicon $\mathrm{L}_{2,3}$-edge was calculated using the ICXANES program of Vvedensky et al. [3], initially with the silicon atom tetrahedrally coordinated to a single shell of oxygen atoms. To investigate the effect of the metal cation on the result, calculations were also performed including the second coordination sphere. Phase shifts were obtained using Hartree-Fock freeatom wavefunctions and a muffin tin potential imposed on the crystal structure. Transitions to both d-like and s-like final states were included in the calculation, however, no interference between the two excitation channels was considered. A similar approach was used to calculate the contribution due to final states with p-like symmetry. Owing to the arbitrary position of the muffin tin energy zero all theoretical energy scales are relative, and the standard procedure is to simply align the first peak in the theoretical curve with the corresponding feature in the experimental spectrum.

Initially, consider the XANES calculations for a perfect $\mathrm{SiO}_{4}$ tetrahedron with no correction for the effect of the core-hole (Fig. 3a). The spectrum consists of three major peaks, B, C and $\mathrm{D}$, which correspond closely to the peaks labelled $\beta, \gamma$ and $\delta$ in the experimental spectra. There 
is no evidence for a pre-peak, and the first major peak results from transitions to both s-like and d-like empty states. Increasing the number of shells in the cluster - simulating the structure of $\alpha-\mathrm{SiO}_{2}$ - has almost no effect on the resultant calculation suggesting that the dominant contribution arises from the first coordination shell. When a core hole is incorporated into the calculations via a $(Z+1)^{*}$ approximation [7] for the central atom in the cluster, significant changes are observed (Fig. 3b). Four distinct peaks, which have energy separations in reasonable agreement with those observed experimentally, are now visible. The first peak, A, is found to be almost entirely a result of transitions to empty states with s-like symmetry, and therefore it is concluded that this corresponds to the state labelled $6 \mathrm{a}_{1}$ in the MO calculation. Peaks B and C in figure $3 \mathrm{~b}$ are primarily due to states with d-like symmetry, although there is some contribution to B from states with s-like symmetry. Comparison of the relative intensities of peaks A and B with those of the first two peaks in the experimental data suggests that there is another contribution to peak $B$. This is confirmed in figure $3 c$, where the result of the XANES calculation of the $p \rightarrow p$ transition is displayed. This calculation clearly indicates that the major contribution to peak $\beta$ in the experimental spectrum arises from this transition. Thus, the four peaks observed in the energy loss spectra are designated as: $\alpha-6 \mathrm{a}_{1}, \beta-6 \mathrm{t}_{2}$, and $\gamma-\left\{2 \mathrm{e}, 7 \mathrm{t}_{2}\right\}$. This is in agreement with the assignments by Dehmer [16] and Bianconi [17] for $\mathrm{x}$-ray absorption spectra provided some contribution to the $6 \mathrm{t}_{2}$ MO from states with s- and d-like symmetry is acknowledged. The XANES calculations indicate that there is no significant contribution to peak $\gamma$ from a state with s-like symmetry. Based on the XANES calculations, peak $\delta$ in the energy loss spectra is primarily associated with states of d-like symmetry.

Previous determinations of the conduction band threshold in the $\mathrm{Si}_{2,3}$-edge of $\alpha-\mathrm{SiO}_{2}$ have placed it between the experimentally observed peaks $\alpha$ and $\beta[17,19]$. Assuming this is also the case in the nesosilicates studied, this implies that peak $\alpha$ corresponds to transitions to a bound state which has been pulled down below the bottom of the conduction band by the core hole potential. Peaks $\beta, \gamma$ and $\delta$ then correspond to quasibound states embedded in the continuum. Figure $3 \mathrm{~d}$ shows the result of a linear combination of the two XANES calculations (Figs.3b and 3c) to illustrate the success with which the experimental spectrum may be modelled using this approach. A failure of the XANES approach to modelling EELS data is its inability to accurately reproduce the intensity ratios found experimentally. Presumably this is due to the muffin tin form of the potential employed in the calculation which is a poor approximation in relatively open covalent structures which possess directional bonding e.g. silicates.

This analysis provides a satisfactory explanation of the spectra for Fayalite and Willemite, and, to a first approximation, that for Phenacite. However, the XANES calculations of a perfect $\mathrm{SiO}_{4}$ tetrahedron clearly cannot be used to interpret the experimental data for Forsterite. The source of the extra intensity observed in the $\mathrm{L}_{2,3}$-edge of Forsterite lies in the distortion of the silicate tetrahedra in this material. For regular tetrahedra, the angular distortions are conveniently represented by the variance of the central angle which, for the samples studied here, has been calculated [18] from the single crystal XRD information as $49.4 \mathrm{deg}^{2}$ (Forsterite), $36.7 \mathrm{deg}^{2}$ (Fayalite), 3.81 $\operatorname{deg}^{2}$ (Phenacite) and $3.29 \mathrm{deg}^{2}$ (Willemite); in Forsterite, the $\mathrm{SiO}_{4}$ tetrahedron is more distorted than in any of the other materials studied.

To incorporate the distortion present in the $\mathrm{SiO}_{4}$ unit of Forsterite, the XANES calculation was repeated using the crystallographic data for the mineral. In this way, distortion of the silicate tetrahedron is directly accounted for in the computation. The calculation was performed using a $(Z+1)^{*}$ approximation to account for the core hole, and the results (Fig. 4) give some indication of the source of the extra intensity in the $\mathrm{Si}_{2,3}$-edge of Forsterite. The number of peaks and their energies in figure $4 \mathrm{a}$ are similar to those in figure $3 \mathrm{~b}$. However, the separation of the maxima of $\mathrm{A}$ and $B$ has increased, and consequently a broad band of intensity is observed just above the edge onset. This is even more apparent in the calculation of the contribution due to $p \rightarrow p$ transitions 


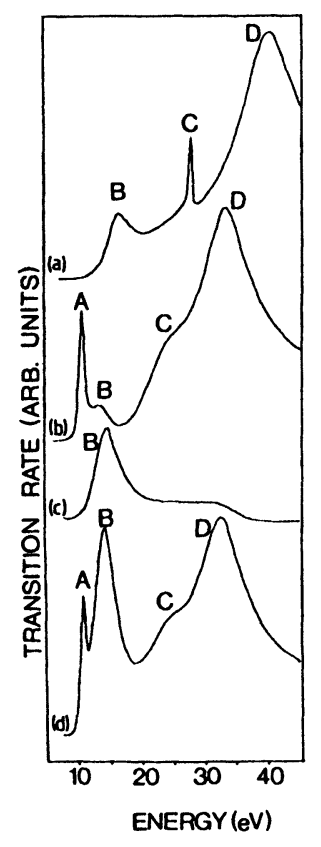

Fig. 3. - Plots showing the results from the XANES calculations for a perfect $\mathrm{SiO}_{4}$ tetrahedron. (a) shows the total intensity due to final states with s- and d-like symmetry, and (b) shows the same result after inclusion of a core-hole via the $(Z+1)^{*}$ approximation. The contribution due to final states with p-like symmetry, including the effect of the core hole, is shown in (c), and in (d) a linear combination of the XANES calculations is shown for comparison with the experimental data.

(Fig. 4b), where distortion has resulted in the intensity due to this transition being split into two distinct peaks. Thus, distortion of the tetrahedron causes the contribution of transitions to the $6 t_{2}$ final state to be split, and results in the filling-in of the valley indicated as $\beta^{\prime}$ in the spectra from non-distorted tetrahedra. The final result is consistent with the experimental data for Forsterite, and indicates that the change in the observed ELNES is primarily caused by geometric effects. To confirm this, calculations were performed in which a second coordination sphere containing the metal cations was included. If the Forsterite spectrum was different to that of Fayalite because of electronic effects, a XANES computation in which the magnesium atoms were incorporated would be expected to highlight the differences. Inclusion of the second coordination sphere in such a calculation was found to have no effect, providing further support for an explanation based on geometrical considerations.

Next consider the experimental spectrum of Phenacite. While the peaks observed can be interpreted to some extent using the MO approach described above, the energy difference and intensity ratio of the first two peaks in the spectrum differ significantly from those found for the isostructural material, Willemite. The explanation of this difference cannot be distortion of the silicate tetrahedra, since the angular variances of the materials are very small. To understand the spectrum of Phenacite, it is necessary to return to the earlier observation that the energy of the $6 a_{1}$ state in the MO diagram is highly sensitive to electronic effects. In Phenacite, the cation is $\mathrm{Be}^{2+}$. This is a very small, highly polarizing ion, and it is suggested that its presence results in a modification of the electron density in the $\mathrm{SiO}_{4}$ tetrahedron and thus causes the conduction band threshold to shift to higher energies due to a more positive effective charge on the silicon atom. 


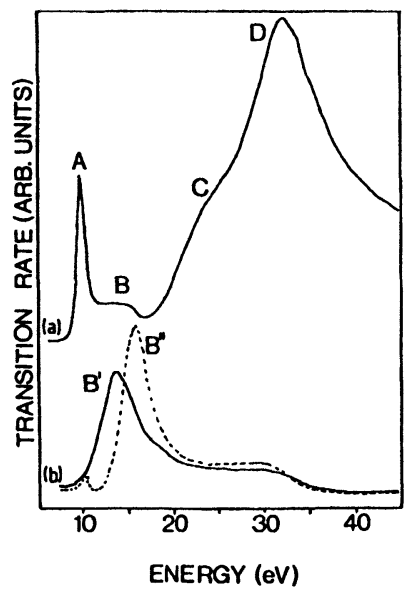

Fig. 4. - Plots showing the results from the XANES calculations for a distorted $\mathrm{SiO}_{4}$ tetrahedron such as that found in Forsterite. (a) shows the total intensity due to final states with s-and d-like symmetry, and (b) displays the contribution due to final states with p-like symmetry. The effect of a core hole has been included in both calculations. Note the broadening of peak B in (a) (compare with Fig. 3b) and the splitting of peak $B$ in figure $3 c$ into two peaks $\mathbf{B}^{\prime}$ and $\mathrm{B}^{\prime \prime}$ in (b), which are associated with final states with quantum numbers $m=0$ and $m=1$, respectively.

This would cause a corresponding shift to higher energy of the bound state, $6 \mathrm{a}_{1}$. As the remaining quasibound states are less sensitive to such changes, the overall result would be to decrease the separation between the $6 \mathrm{a}_{1}$ and $6 \mathrm{t}_{2}$ molecular orbitals, and cause the alteration in the relative peak intensities observed.

\section{Conclusions.}

The energy-loss spectra from nominally identical $\mathrm{SiO}_{4}$ tetrahedra have been shown to exhibit significant differences; an observation which might indicate that a simple coordination fingerprint does not exist in EELS. Analysis of the spectra by comparison with MO diagrams and x-ray absorption results does not lead to an unambiguous interpretation of the data. More importantly, neither approach permits an explanation of the changes in the energy-loss spectra to be achieved.

The spectra have been modelled using the established multiple scattering (XANES) method. In this way, it has been possible to assign each peak in the spectra to empty states of particular symmetry, and to illustrate the importance of considering the effect of the core hole on the ordering of the empty molecular orbitals.

In the case of Forsterite, the energy loss spectrum shows a broad band of intensity which is not present in the spectra of the other nesosilicates which the XANES calculations clearly show to be caused by a splitting of the empty p- and d-like states due to distortion of the tetrahedron. Since the inclusion of a second coordination sphere in the calculations has little effect on their outcome, it is concluded that the abnormalities in the spectrum of Forsterite are primarily a result of geometric effects. Thus, it could be argued that the $\mathrm{SiO}_{4}$ tetrahedron in Forsterite does not exhibit the expected EELS coordination fingerprint. However, because of distortions, the tetrahedron is not regular, and hence the MO diagram calculated for a perfect $\mathrm{SiO}_{4}$ unit is of little use in trying to interpret the energy-loss spectrum of this material. The possibility that electronic effects can 
cause changes in the shape of the silicon $\mathrm{L}_{2,3}$-edge is not discounted; in future publications [20] it will be shown that electronic effects can dominate the ELNES in certain nesosilicates.

The energy-loss spectrum of Phenacite has also been discussed, to demonstrate that it is not sufficient to consider only geometric effects in these analyses. In this mineral, the changes in the energy-loss spectrum, with respect to that of the isostructural material Willemite, are postulated to be caused by the presence of a small, highly polarizing cation, $\mathrm{Be}^{2+}$. The cation causes a shift of the photoconduction threshold to higher energy, and thus the separation of the first two peaks in the energy-loss spectrum decreases and the ratio of their intensities is changed.

In conclusion, the concept of a coordination fingerprint in EELS has been neither proved, nor disproved, but it has been clearly shown that it can be misleading to assume the presence of a particular fingerprint without a clear understanding of all of the factors affecting the structure of the species under investigation.

\section{Acknowledgements.}

We thank Ole Johnsen from the Mineralogical Museum, Copenhagen for supplying the mineral samples. We also thank the Royal Commission for the 1851 Exhibition (DWM) and the Royal Society (RB) for supporting this research, and the Danish Council for Technical Research for funding during a one year visit to the Cavendish Laboratory (PLH). We are grateful to Dr Phil Gaskell, Prof Mick Brown and Prof Archie Howie for many useful discussions about this work.

\section{References}

[1] EgERTON R.F., Electron Energy Loss Spectroscopy in the Electron Microscope (Plenum Press, New York, 1986).

[2] BRYDSON R., EMSA Bulletin "Fall Edition" (1991 - in press).

[3] Vvedensky D.D., SAlding D.K. and PendRY J.B., Computer Phys. Commun. 40 (1986) 421.

[4] Egerton R.F. and Whelan M.J., Philos. Mag. 30 (1986) 739.

[5] Weng X., Rez P. and SANkey O.F., Phys. Rev. B 40 (1989) 5694.

[6] Engel W., SaUer H., Zeitler E., Brydson R., Williams B.G. and ThOMas J.M., J. Chem. Soc. Faraday Trans. I 84 (1988) 617.

[7] Brydson R., Sauer H., Engel W., Thomas J.M. and Zeitler E., J. Chem. Soc. Chem. Commun. (1989) 1010.

[8] TosSEl J.A., J. Am. Chem. Soc. 97 (1975) 4840.

[9] SKIFF W.M., CARPENTER R.W. and LIN S.H., J. Appl. Phys. 58 (1991) 3463.

[10] HansEn P.L., FALlON P.J. and KRÄTSCHMER W., J. Chem. Phys. Lett. 181 (1991) 367.

[11] GRISCOM D.L., J. Non-Cryst. Solids 24 (1977) 155.

[12] BATSON P.E., KAVANAGH K.L., WONG C.Y. and WOODALl J.M., Ultramicroscopy 22 (1987) 89.

[13] Colliex C., Manoubi T., Gasgnier M. and Brown L.M., Scanning Electron Microscopy 11 (1985) 489.

[14] PAYNe R.S., CRICK R.A. and McComb D.W., Proc. of EMAG '91, Inst. Phys. Conf. Ser. (1991, in press).

[15] McMullan D., Rodenburg J.M., Murooka Y. and MCGibbon A.J., Inst. Phys. Conf. Ser. 98 (1989) 55.

[16] DeHMER J.L., J. Chem. Phys. 56 (1972) 4496.

[17] BIANCONI A., Surf. Sci. 89 (1979) 41.

[18] SMYTH J.R. and BISH D.L., Crystal Structures and Cation Sites of the Rock-Forming Minerals (Allen \& Unwin, Boston, 1988).

[19] O'Brien W.L., Jia J., DONG Q.-Y., Calloot T.A., Rubenson J.E., Mueller D.L. and Edererer D.L., Phys. Rev. B 44 (1991) 1013.

[20] MCCOMB D.W., PAYNE R.S., BRYDSON R. and HANSEN P.L., An EELS study of Zircon (in preparation). [21] ATKIns P.W., Physical Chemistry, OUP, Oxford (1983) pp. 545-546. 